\title{
REVISIONES
}

\section{Importancia de la domesticación en la conservación de los hongos silvestres comestibles en México}

\author{
The importance of domestication in the conservation of edible wild fungi in Mexico
}

\author{
Gerardo Alvarado-Castillo ${ }^{\text {a* }}$, Gerardo Mata ${ }^{\text {b }}$, Griselda Benítez-Badillo ${ }^{\text {b }}$ \\ *Autor de correspondencia: a Universidad Veracruzana, Facultad de Ciencias Agrícolas, Posgrado en Ciencias Agropecuarias, \\ Circuito Aguirre Beltrán s/n Zona Universitaria CP. 91090, Veracruz, México, tel.: 52228842 1749, \\ gerardoalvaradoc@hotmail.com \\ b Instituto de Ecología A.C. Km 2.5 Carretera antigua a Coatepec 351, Congregación El Haya, Xalapa, Veracruz, México.
}

\begin{abstract}
SUMMARY
In Latin America, including Mexico, the tremendous degradation of forests has drastically altered the ecological, social and cultural contexts associated with them. Edible wild fungi form part of the eating and subsistence strategy of the local inhabitants and of the biocultural richness. This resource is currently at risk owing to the growing demand for its gastronomic and nutraceutical properties, and because of the lack of knowledge for its sustainable use. All of this increases the probability of overexploiting or driving some species extinct, especially the most traditionally used and well-known ones. Because of this, it is necessary to develop an understanding of the role of edible wild fungi in agroecosystems, and to develop technologies that allow them to be produced under domestication. This review examines the theoretical and conceptual background of the challenges of managing fungi and the implications of their domestication; emphasizing the need to take into account ethnomycological knowledge and use an interdisciplinary approach that favors a balance between conservation and development. The proper management of this resource could contribute to local economies and even become a driver of social transformation for rural and indigenous communities in the forests of Mexico.
\end{abstract}

Key words: agroecosystems, forest ecosystems, traditional knowledge, ethnomycology.

\section{RESUMEN}

En Latinoamérica, incluido México, existe una gran degradación de los bosques y selvas que ha alterado drásticamente al entorno ecológico, social y cultural asociado a ellos. Los hongos silvestres comestibles forman parte de esta riqueza biocultural y se encuentran actualmente amenazados por el desconocimiento de pautas de aprovechamiento sostenible y la creciente demanda por sus propiedades gastronómicas y nutracéuticas. Esto aumenta la probabilidad de sobreexplotación o extinción de distintas especies, especialmente de aquellas tradicionales y más conocidas. Por lo anterior, es necesario entender el papel de los hongos silvestres comestibles dentro de los agroecosistemas, así como desarrollar tecnologías que permitan su domesticación y producción. En la presente revisión se analizan antecedentes teóricos y conceptuales relacionados con los retos en el manejo del recurso fúngico y las implicancias de su domesticación, señalando la necesidad de considerar el conocimiento etnomicológico y un enfoque interdisciplinario que favorezca el equilibrio entre conservación y desarrollo. El manejo adecuado de este recurso podría contribuir a las economías locales y ser un factor de transformación social para las comunidades rurales e indígenas de los bosques en México.

Palabras clave: agroecosistemas, ecosistemas forestales, conocimiento tradicional, etnomicología.

\section{INTRODUCCIÓN}

Los hongos silvestres comestibles junto con otros productos forestales no maderables (PFNM) (alimento, medicina, materiales de construcción, leña, etc.), constituyen un elemento relevante en la alimentación e ingresos de varios millones de hogares en todo el mundo, por lo que gobiernos e instituciones han comenzado a valorar su importancia dentro de las comunidades rurales (Zamora-Martínez y Nieto de Pascual-Pola 1995, Mariaca et al. 2001), por su aporte a la autosuficiencia alimentaria y obtención de ingresos (Pastor 2002, Pérez-Moreno et al. 2010).

En México su recolección es una actividad basada en el uso múltiple de los recursos naturales, que junto con otras actividades tales como la siembra de maíz, cría de animales, cacería ocasional y venta de la propia fuerza de trabajo, forma parte de la estrategia alimentaria y de subsistencia de los productores rurales que habitan las zonas 
boscosas del país (Tacón y Palma 2006, Estrada-Martínez et al. 2009). Esta actividad se ha realizado desde épocas prehispánicas y está integrada por componentes económicos, sociales, culturales y ecológicos, lo que le otorga el carácter de agroecosistema, ya que consta de elementos ambientales y humanos, que poseen forma, dinámica, función e interrelaciones (Alvarado y Benítez 2009), producto de un proceso eminentemente histórico, cultural y natural (Ruan-Soto et al. 2013).

Económicamente, es una de las pocas actividades que permiten generar un ingreso adicional en comunidades marginales, ya que no demanda de inversiones ni herramientas e incluye la participación de todos los miembros de la familia, lo que permite asegurar un flujo monetario temporal y complementario en muchos hogares rurales (Mariaca et al. 2001, Estrada-Martínez et al. 2009, PérezMoreno et al. 2010), sobre todo en la época de baja actividad agrícola (Montoya et al. 2008). Posee un componente social muy arraigado que genera vínculos entre familias y comunidades (Villareal y Pérez-Moreno 1989, Pastor 2002), con un gran impacto de género, pues las mujeres se encuentran involucradas en la recolección, procesamiento y particularmente en la comercialización (Mariaca et al. 2001, Pérez-Moreno et al. 2008).

Incluye importantes elementos culturales determinados por el género, origen, ocupación y grupo étnico de la población rural, así como una extensa comprensión sobre su ingestión, propiedades medicinales y uso ritual (Guzmán 1994, Garibay-Orijel et al. 2012, Ruan-Soto et al. 2013). Estos conocimientos ancestrales se mantienen activos de manera vertical (de padres a hijos) y horizontal (entre gente de la misma generación) (Mariaca et al. 2001, Estrada-Martínez et al. 2009), conformando parte de su identidad cultural (Garibay-Orijel et al. 2012) y manteniendo dicho conocimiento hasta el día de hoy (Pérez-Moreno et al. 2008).

La recolección de hongos silvestres comestibles es compatible con la conservación de los recursos naturales, ya que mantiene una producción continua de los bienes y servicios que proporciona el bosque (Villareal y PérezMoreno 1989). Forma parte de su ciclo y contribuye a su salud y productividad (Bonet et al. 2012, Castillo-Guevara et al. 2012, Montoya y Orrego 2012), conformando con ello un componente ecológico, pues este tipo de aprovechamiento ocasiona menos impactos que otras actividades como la ganadería (Wilsey 2006), generando en las comunidades un incentivo para proteger sus recursos forestales.

De esta forma, la diversidad étnica y de recursos bióticos existente en las diferentes regiones agroecológicas de México, permite que se realice el aprovechamiento de los hongos silvestres comestibles con una visión particular y distinta, que conforma la estrategia de supervivencia de muchos grupos rurales e indígenas (Solbrig 2004). En tal sentido, se mantiene el conocimiento empírico de un recurso importante, que aporta ingresos, beneficia la conservación del ambiente y promueve la organización social, usos y costumbres de las comunidades establecidas en los ecosistemas forestales (Alvarado y Benítez 2009). Todo lo cual forma parte de la riqueza biocultural de México, que ha representado una importante forma de conservación del recurso in situ (Hernández 1993).

Por lo anterior, el objetivo de la presente revisión es informar y difundir la importancia de la recolección de hongos silvestres comestibles en México, los factores que la integran y las limitantes a las que se enfrenta, planteando a la domesticación como un eje fundamental de la conservación del recurso.

\section{SITUACIÓN ACTUAL Y RETOS EN EL MANEJO DE LOS HONGOS SILVESTRES COMESTIBLES}

En México, la recolección de hongos silvestres comestibles es una de las actividades más aisladas y poco conocidas del sector primario (Villareal y Pérez-Moreno 1989, Arteaga y Moreno 2006), pues se desconocen sus patrones de desarrollo, estructuras, procesos, variables socioeconómicas e interrelaciones con otros sectores (Mariaca et al. 2001, Martínez-Carrera et al. 2007, Garibay-Orijel et al. 2009). Así mismo, el alto valor de algunos de ellos en los mercados nacionales e internacionales (por ejemplo, especies de los géneros Morchella, Tricholoma y Boletus) junto con los vacíos legales existentes, pueden desplazar a los recolectores tradicionales, quienes usan normalmente este recurso como estrategia de subsistencia, por personas contratadas para recolectar grandes volúmenes, lo cual generalmente se realiza de forma desigual y desordenada, al acceder a tierras comunales y federales sin los permisos correspondientes y arrasar con las especies comerciales.

Este saqueo, resultado de una falta de gestión forestal sostenible, provoca impactos como la perturbación excesiva del hábitat, compactación del suelo (Martínez de Aragón et al. 2011), interrupción del ciclo biológico de algunas especies, fluctuación y disminución de su abundancia, erosión genética (Tacón y Palma 2006), etc., que interfieren en el importante papel que juegan los hongos en la salud forestal (Bonet et al. 2012, Montoya y Orrego 2012).

Políticas públicas en el aprovechamiento de los hongos silvestres comestibles. En México, los hongos (cultivados, silvestres y medicinales) no figuran en los sistemas de información oficiales (los cuales determinan la relevancia social y la jerarquización de prioridades en las actividades productivas), por lo que existe una ausencia de políticas públicas, estrategias de mercado y fomento a la investigación científica en torno a este recurso. Ejemplo de ello son las áreas de oportunidad desaprovechadas en hongos silvestres y medicinales con potencial de exportación. Adicionalmente, el marco legal que regula el aprovechamiento de los hongos silvestres es muy general y relega sus competencias a diversas leyes y ordenamientos, lo que ocasiona que este sea ambiguo y genere confusión o duplicidad (Benítez-Badillo et al. 2013). 
Así mismo, existe un problema de tenencia de la tierra, donde el acceso al bosque no tiene ninguna restricción y la propiedad de los PFNM no está claramente asignada, por lo que los ejidos, usufructuarios y concesionarios de tierras públicas no reciben compensación o beneficio alguno, desincentivando la conservación del bosque (Martínez de Aragón et al. 2011). A lo anterior se añade el escaso interés público y privado para estimular la producción, y los problemas técnicos de almacenamiento, conservación y transporte (Martínez-Carrera et al. 2007, Andrade et al. 2012). El desconocimiento del potencial de este recurso, favorece los procesos de conversión de los ambientes de bosque o selva para otros usos productivos, generalmente más severos, intensivos o incompatibles, generando deterioro y alteraciones no sólo a las poblaciones fúngicas, sino también al entorno social y cultural (Estrada-Martínez et al. 2009).

El papel de los hongos silvestres comestibles en la gestión forestal sostenible. La Conferencia Ministerial de Helsinki (1993) declaró que la gestión forestal sostenible es la "administración y uso de los bosques y los montes de manera y en tal medida que mantengan su biodiversidad, productividad, capacidad de regeneración, vitalidad y su potencial de cumplir, ahora y en el futuro, funciones ecológicas, económicas y sociales relevantes, a escala local, nacional y global, sin causar daño a otros ecosistemas“. En ese aspecto los hongos desempeñan un papel clave, pues contribuyen al reciclamiento de nutrientes, a través de la descomposición de residuos lignocelulósicos y excretas de animales, al manteniendo la fertilidad del suelo (Savoie y Largeteau 2011), forman parte de la cadena trófica y mantienen interacciones con la flora y fauna, contribuyendo a la salud del sistema forestal (Bonet et al. 2012, Montoya y Orrego 2012), aunque lamentablemente su importancia aún no se percibe como tal.

Dentro de los hongos silvestres comestibles más importantes se encuentran los micorrícicos, puesto que aproximadamente el $80 \%$ de las plantas pertenecen a familias capaces de formar asociaciones simbióticas con ellos. Estos mejoran la nutrición del árbol (Boa 2004, Egli 2011, Savoie y Largeteau 2011), a través de la asimilación de elementos poco móviles como nitrógeno, fósforo, cobre y zinc (Martínez-Peña et al. 2012) y otorgan mayor tolerancia al estrés ambiental, nutrimental y a factores externos (ataque de patógenos o insectos) (Smith y Read 2008, Sangabriel-Conde et al. 2010). Pueden ser utilizados como bioinoculantes para micorrización artificial (Savoie y Largeteau 2011) tal como se ha utilizado en México Amanita caesarea (Scop) Pers. para Pinus patula Schl. et Cham. y Laccaria laccata (Scop.) Cooke para P. greegii Englem., P. patula y P. pseudostrobus Lindl. (Jiménez et al. 2013).

Adicionalmente, los hongos sirven de alimento a la fauna local, la cual a su vez contribuye a su dispersión y a la regeneración vegetal en zonas perturbadas, dado que las excretas son excelente fuente de inoculo micorrizógeno
(Castillo-Guevara et al. 2012). Todo lo anterior favorece el equilibrio de los ciclos biogeoquímicos (Savoie y Largeteau 2011, Martínez-Peña et al. 2012), fomenta la productividad y contribuye a la resiliencia de los bosques (Savoie y Largeteau 2011).

Integrar el conocimiento de los diversos roles de los hongos en el funcionamiento del ecosistema, junto con el saber tradicional de los pueblos que habitan esos ambientes, puede orientar el manejo integral del bosque (Egli 2011, Savoie y Largeteau 2011), por lo que en la actualidad, el aprovechamiento racional de los hongos silvestres comestibles y su conservación a largo plazo necesitan de la gestión de las poblaciones naturales, basados en el conocimiento local y el desarrollo de técnicas para su domesticación. Esta puede conducir a un nuevo enfoque de conservación y aprovechamiento sostenible, constituyendo una respuesta ante el estado de vulnerabilidad de muchas especies en su medio natural (Ruiz et al. 2004, Andrade et al. 2012), lo cual hace necesario analizar los antecedentes teóricos y conceptuales que permitan dilucidar la viabilidad de esta opción en el futuro.

\section{LA DOMESTICACIÓN: ANÁLISIS DEL CONCEPTO}

El tema se encuentra en amplia discusión, pues existe confusión con otros términos como cultivo y producción, además de que su delimitación no es fácil ya que es un proceso complejo e intangible. Convencionalmente se refiere a un proceso por medio del cual plantas, animales y microorganismos son extraídos de su medio natural para adaptarlos a hábitats creados por el hombre con fines de reproducción y consumo directo o indirecto. Este lleva implícito un proceso evolutivo como resultado de la selección y cruzamiento de una especie, así como el manejo de su entorno natural, para la obtención de características deseables, de tal manera que estas modificaciones genéticas sean acumuladas y heredadas a través del tiempo (Solbrig 2004) para la obtención de fenotipos culturalmente deseados (Abbo et al. 2012). Sin embargo, esta selección y manejo implica eliminar o retener ciertas características, conduciendo así a una alta uniformidad morfogenética, lo que supone un riesgo ante epidemias y enfermedades (Hernández 1993), así como una dependencia de la intervención humana (Krapovickas 2011).

No obstante, existen otros conceptos que indican que la domesticación va más allá de la definición biológica convencional e integra aspectos culturales, entendiéndola como un proceso evolutivo en el tiempo, con avances, grados y estadíos intermedios (Krapovickas 2011), en el que intervienen las actividades humanas, los procesos físicos del medio y los intercambios mutuos, todos incluidos en un solo sistema analítico. Esto indica que la domesticación implica una serie de interrelaciones simbióticas y co-evolutivas entre el hombre y el ambiente, que a su vez dependen del desarrollo cultural de los pueblos que la practican (Hernández 1993, Jakuba et al. 2008). 
Con los elementos expuestos, el concepto convencional de domesticación en los hongos silvestres comestibles puede complementarse, pues integra aspectos sociales, culturales y biológicos, que se han desarrollado en diferentes direcciones y en varios niveles de organización (individual, ecosistema, comunidad) a través del tiempo y no únicamente en respuesta a presiones de subsistencia de los grupos sociales (alimentación), sino como un proceso social y simbólico en el que la naturaleza es integrada a un sistema cultural (Bell y Walker 1992). La influencia de todos estos factores (ecológicos, evolutivos, culturales y tecnológicos) hacen que sea un proceso dinámico y en continuo cambio (Meyer et al. 2012), aún en proceso de construcción.

\section{EL PROCESO DE DOMESTICACIÓN Y SU POTENCIAL EN LOS HONGOS SILVESTRES COMESTIBLES}

Se han estimado a nivel mundial 1,5 millones de especies de hongos, los cuales se clasifican según su tamaño en micromicetos y macromicetos (Chang y Miles 2004). Dentro de los primeros, se considera a las levaduras como los primeros en ser domesticados para la elaboración de quesos, cerveza u otros productos alimenticios, ocupando en la actualidad lugares importantes dentro de procesos biotecnológicos (Solbrig 2004). Otro ejemplo es la utilización de Penicillium spp. en la medicina y de Saccharomyces spp. en la elaboración de bioenergéticos, aplicación que se vislumbra como una de las más prometedoras a futuro.

En cuanto a los macromicetos u hongos superiores, existen aproximadamente 10.000 especies que producen cuerpos fructíferos, pero sólo 2.000 (pertenecientes a 31 géneros) son consideradas comestibles. De esta cifra, alrededor de 100 se cultivan experimentalmente, 50 poseen valor económico y sólo 30 son comercialmente cultivadas (Kües y Liu 2000, Chang y Miles 2004). No obstante, Martínez-Carrera et al. (2007) mencionan que únicamente 10 especies se cultivan a nivel industrial, mientras Chang y Miles (2004) indican que son solo seis.

En México, Guzmán (1994) estima que hay más de 200.000 especies, pero sólo 4 \% son conocidas, aunque es el país de Latinoamérica con mayor cantidad de hongos silvestres comestibles registrados (Andrade et al. 2012) con más de 300 variedades (Burrola-Aguilar et al. 2012). El potencial de domesticación de los hongos silvestres comestibles (en el sentido convencional) está limitado por el escaso conocimiento de su ciclo de vida e interrelaciones ecológicas, por lo que se siguen obteniendo mediante recolección (Savoie y Largeteau 2011). Esto implica constantes variaciones en los volúmenes colectados en su medio natural y un alto valor de mercado.

Esta situación ha motivado la necesidad de investigar su cultivo y generar los principios para su domesticación. $\mathrm{Al}$ respecto, diversos estudios han demostrado que es posible obtener hongos en condiciones naturales, a través de su inoculación directa en campo o con el uso de plantas micorrizadas (Kües y Liu 2000, Morcillo y Sánchez 2004, Jiménez et al. 2013), destacando los trabajos realizados en diferentes especies de trufas (Tuber melanosporum Vittad, T. uncinatum Chatin, T. borchii Vittad) y sus mecanismos de establecimiento (Wang y Hall 2004, Reyna 2012a, Reyna y Garcia-Barreda 2014), así como algunos avances modestos en la producción de Tricholoma magnivelare (Peck) Redhead, T. matsutake (S. Ito et S. Imai) Singer (Iwase 1997) y Cantharellus cibarius Fr. (Danell y Camacho 1997).

Lo anterior muestra que la domesticación y cultivo de hongos silvestres, (especialmente micorrícicos) es posible y puede darse a escala comercial (Kües y Liu 2000), siempre y cuando se considere al bosque como una parte integral de este proceso, como se ha demostrado en la implementación de técnicas de producción de trufas (Reyna 2012b), esto, con el descubrimiento de propiedades medicinales y nutracéuticas, aumentan cada día el interés en la investigación y desarrollo tecnológico para la implementación de la domesticación de los hongos (Sánchez y Mata 2012).

Especies con potencial de domesticación. Aunque existen avances en la domesticación convencional de géneros saprófitos, como Lepista, Lentinula, Ganoderma y Agaricus (Mata y Savoie 2012, Sánchez y Mata 2012), las especies micorrícicas, por su condición, tienen que optar por otro tipo de esquema, cuyo potencial de manejo está estrechamente relacionado con la diversidad de especies forestales. En este sentido, México cuenta con más de 64 millones de hectáreas de superficie forestal ( $47 \%$ del total nacional) que incluye bosques (coníferas $=5,64 \%$, coníferas y latifoliadas $=9,33 \%$, latifoliadas $=8,05 \%$, bosque mesófilo de montaña $=1,23 \%$ ), selvas (altas-medianas $=10,19 \%$, bajas $=$ $11,50 \%)$, manglares $(0,42 \%)$ y otras asociaciones $(0,4 \%)$, de las cuales 52,94 \% está caracterizada como vegetación primaria (Sistema Nacional de Información Forestal 2012). Esta variedad de ecosistemas, favorecidos por la ubicación, complejidad orográfica, climática y geológica de los bosques y selvas de México, genera una gran diversidad fúngica (Aguilar-Cruz y Villegas 2010, Burrola-Aguilar et al. 2013). Para ilustrarlo, el cuadro 1 indica algunos géneros de hongos y su compatibilidad con varios géneros forestales.

Además de esta gran biodiversidad, las comunidades rurales de las distintas áreas de México, poseen una profunda comprensión sobre las propiedades, taxonomía, biología y ecología local de muchos de los hongos relacionados a los ecosistemas forestales (Garibay-Orijel et al. 2012, Ruan-Soto et al. 2013), prueba de ello es la nomenclatura tradicional que hace referencia a su morfología, color, lugar de crecimiento e incluso a los árboles asociados (Ruan-Soto et al. 2009, Jiménez et al. 2013, Lara-Vázquez et al. 2013). Este conocimiento puede dar lugar a un proceso de domesticación a través de la integración de términos sociales, culturales y biológicos, para un manejo acorde a las condiciones de cada región en particular o a través 
Cuadro 1. Géneros de hongos y árboles capaces de formar asociaciones micorrícicas.

Genera of fungi and trees able to form mycorrhizal associations.

\begin{tabular}{|c|c|}
\hline Género de hongos & Géneros forestales asociados \\
\hline Amanita & $\begin{array}{l}\text { Abies, Acacia, Afzelia, Aldinia, Allocasuarina, Alnus, Anisoptera, Anthonota, Aphanocalyx, Berlinia, Betula, } \\
\text { Brachystegia, Casuarina, Corylus, Dicymbe, Didolotia, Dipterocarpus, Dryobalanopus, Eperua, Eucalyptus, } \\
\text { Fagus, Gilbertiodendron, Hopea, Inga, Instia, Isoberlinia, Julbernardia, Larix, Leptolaena, Macrolobium, } \\
\text { Marquesia, Microberlinia, Monopetalanthus, Monotes, Ormosia, Paraberlinia, Paramacrolobium, } \\
\text { Pericopsis, Picea, Pinus, Populus, Pseudotsuga, Quercus, Salix, Sarcolaena, Schizolaena, Shorea, } \\
\text { Tetraberlinia, Tsuga, Uapaca y Vateria. }\end{array}$ \\
\hline Boletus & $\begin{array}{l}\text { Abies, Alnus, Anisoptera, Betula, Castaneopsis, Corylus, Dipterocarpus, Dryobalanopu, Eucalyptus, Fagus, } \\
\text { Hopea, Larix, Lithocarpus, Marquesia, Monotes, Pasania, Picea, Pinus, Populus, Pseudotsuga, Quercus, } \\
\text { Salix, Shorea, Tsuga y Vateria. }\end{array}$ \\
\hline Cantharellus & $\begin{array}{l}\text { Abies, Acacia, Afzelia, Aldinia, Alnus, Anisoptera, Anthonota, Aphanocalyx, Berlinia, Betula, Brachystegia, } \\
\text { Coccoloba, Corylus, Dicymbe, Didolotia, Dipterocarpus, Dryobalanopus, Eperua, Eucalyptus, Fagus, } \\
\text { Gilbertiodendron, Hopea, Inga, Instia, Isoberlinia, Julbernardia, Larix, Macrolobium, Marquesia, } \\
\text { Microberlinia, Monopetalanthus, Monotes, Ormosia, Paraberlinia, Paramacrolobium, Pericopsis, Picea, } \\
\text { Pinus, Populus, Pseudotsuga, Quercus, Salix, Shorea, Tetraberlinia, Tsuga, Uapac y Vateria. }\end{array}$ \\
\hline Hydnum & Abies, Pinus y Quercus. \\
\hline Laccaria & $\begin{array}{l}\text { Abies, Allocasuarina, Alnus, Bambusa, Betula, Campomanesia, Casuarina, Corylus, Eucalyptus, Eucalyptus, } \\
\text { Eugenia, Fagus, Larix, Melaleuca, Picea, Pinus, Populus, Pseudotsuga, Quercus, Salix, Tristania y Tsuga. }\end{array}$ \\
\hline Lactarius & $\begin{array}{l}\text { Acacia, Afzelia, Aldinia, Alnus, Anisoptera, Anthonota, Aphanocalyx, Berlinia, Betula, Brachystegia, Corylus, } \\
\text { Dicymbe, Didolotia, Dipterocarpus, Dryobalanopus, Eperua, Eucalyptus, Fagus, Gilbertiodendron, Hopea, } \\
\text { Inga, Instia, Isoberlinia, Julbernardia, Larix, Macrolobium, Marquesia, Microberlinia, Monopetalanthus, } \\
\text { Monotes, Ormosia, Paraberlinia, Paramacrolobium, Pericopsis, Picea, Pinus, Populus, Pseudotsuga, } \\
\text { Quercus, Salix, Shorea, Tetraberlinia, Tsuga, Uapaca y Vateria. }\end{array}$ \\
\hline $\begin{array}{l}\text { Lycoperdon, } \\
\text { Ramaria, } \\
\text { Tricholoma }\end{array}$ & $\begin{array}{l}\text { Abies, Alnus, Betula, Corylus, Eucalyptus, Fagus, Larix, Picea, Pinus, Populus, Pseudotsuga, Quercus, } \\
\text { Salix y Tsuga. }\end{array}$ \\
\hline Rusula & $\begin{array}{l}\text { Acacia, Afzelia, Aldinia, Anisoptera, Anthonota, Aphanocalyx, Berlinia, Brachystegia, Dicymbe, Didolotia, } \\
\text { Dipterocarpus, Dryobalanopus, Eperua, Gilbertiodendron, Hopea, Inga, Instia, Isoberlinia, Julbernardia, } \\
\text { Macrolobium, Marquesia, Microberlinia, Monopetalanthus, Monotes, Ormosia, Paraberlinia, } \\
\text { Paramacrolobium, Pericopsis, Shorea, Tetraberlinia, Uapaca y Vateria. }\end{array}$ \\
\hline угоріич & Us. \\
\hline
\end{tabular}

Fuente: elaborado con datos de Savoie y Largeteau (2011), Pérez-Moreno (2012), Sánchez y Mata (2012) y Jiménez et al. (2013).

del aprovechamiento de esta información para generar modelos predictivos para la identificación, establecimiento y manejo de áreas potenciales.

Conservación forestal y micosilvicultura. El aprovechamiento forestal usualmente se ha centrado en la producción de madera, sin embargo, los altos costos de producción, la globalización (competencia con las importaciones) y las preocupaciones públicas para la protección del ecosistema (Martínez de Aragón et al. 2011) hacen necesario un nuevo enfoque de manejo del bosque. En este sentido, la recolección de hongos ha demostrado una rentabilidad igual o mayor (hasta un 60 \% más) que la producción maderable (Bonet et al. 2010, Martínez de Aragón et al. 2011, Bonet et al. 2012) y la creciente demanda de estos
PFNM por sus propiedades nutracéuticas, han aumentado la importancia relativa de los hongos en el bosque. Por ello es necesario integrar la producción de madera y hongos (cuyo crecimiento y productividad están interconectados) (Fernández-Toirán et al. 2006, Bonet et al. 2010) con su relevancia sociocultural, de tal forma que pueda generar un cambio en el manejo del bosque y sus prácticas, detonando una planeación tradicional, enfocada a un esquema complejo de silvicultura multifuncional, es decir la micosilvicultura (Savoie y Largeteau 2011)

En México, el desarrollo de este esquema es incipiente en comparación con Europa, donde ha cobrado gran relevancia (Savoie y Largeteau 2011) lo que constituye un reto para el aprovechamiento sostenible del bosque, por lo que su gestión, a través de prácticas como la micorrización 
controlada, la inoculación in situ y prácticas de manejo forestal (raleo, aclareo y podas) ofrecen un avance prometedor para la obtención de estos PFNM (Bonet et al. 2012). Esto es de gran importancia, porque el aprovechamiento actual se realiza sobre la base del conocimiento empírico, lo cual limita el manejo sostenible de las poblaciones naturales, por la falta de métodos que permitan pronosticar su producción a partir de variables ecológicas, dasonómicas o fisiológicas. Ante ello es necesario la generación de modelos de predicción que consideren las características locales (altitud, pendiente, orientación) estructura del bosque (densidad, edad, especies presentes) y variables climáticas (precipitación y temperatura) (Fernández-Toirán et al. 2006, Savoie y Largeteau 2011, Martínez-Peña et al. 2012), considerando además la recopilación y sistematización del conocimiento tradicional.

Modelos predictivos para el aprovechamiento de los hongos silvestres comestibles. Estos son una herramienta de la micosilvicultura (generalmente realizados en masas forestales uniformes de Pinus spp.), que han demostrado que el manejo forestal tiene influencia sobre la obtención de hongos, por ejemplo, estudios recientes han podido establecer modelos predictivos de producción de Boletus edulis Bull (Ortega-Martínez y Martínez-Peña 2008, Martínez-Peña et al. 2012) y Lactarius deliciosus (L. ex Fr.) Gray (Bonet et al. 2012, Martínez-Peña et al. 2012), aunque también se han desarrollado investigaciones para la producción de otras especies como Cantharellus cibarius, Lyophyllum shimeji Kawam y Suillus granulatus (L. Fr.) Roussel (Savoie y Largeteau 2011). En estos se identifican los principales factores bióticos y abióticos asociados a la producción de hongos y se ha conseguido simular y establecer itinerarios para un manejo bajo el esquema de micosilvicultura (Bonet et al. 2012).

A pesar del gran progreso que representan estos modelos, existe entre ellos una gran variabilidad asociada a una gran cantidad de factores involucrados, como los climáticos (paradójicamente la precipitación y la temperatura son factores comunes) (Bonet et al. 2012, Martínez-Peña et al. 2012), aspectos relacionados con la ubicación (altitud, orientación o pendiente en la que se encuentra la masa forestal) y aspectos asociados a las características estructurales del bosque (especies de árboles, área basal, edad del rodal, densidad, etc.) (Fernández-Toirán et al. 2006, Bonet et al. 2010, 2012), los cuales además parecen estar interrelacionados entre sí. Esta variabilidad, heterogeneidad y gran número de factores que potencialmente intervienen en la producción de hongos, hacen necesario el uso de técnicas de modelización a nivel local, que ayuden a identificar los aspectos más importantes en la producción y diversidad de los hongos, pues lo que genera condiciones favorables para ciertas especies, puede tener efectos negativos en otras (Bonet et al. 2012).

Aunque la gran cantidad de variables relacionadas con la productividad de los hongos y su interdependencia di- ficulta hacer recomendaciones generales para un manejo micosilvícola, los resultados de estos estudios son alentadores, ya que demuestran que la producción de hongos están influenciadas por el manejo forestal, lo que hace posible generar planes que integren la producción de hongos en los modelos silvícolas (Bonet et al. 2010) lo que junto con el conocimiento tradicional y el monitoreo multianual, pueden generar, para las condiciones de México, una silvicultura de uso múltiple.

\section{LA NECESIDAD DE DOMESTICACIÓN DE LOS HONGOS SILVESTRES COMESTIBLES}

La necesidad de domesticación de los hongos silvestres comestibles se encuentra enmarcada en dos aspectos primordiales: el desconocimiento de la actividad y la creciente demanda de estos productos. El primer caso tiene como consecuencia que se le relegue de los temas prioritarios, se excluya de los esquemas de apoyos, servicios y acciones estratégicas del gobierno y del sector privado (MartínezCarrera et al. 2007, Benítez-Badillo et al. 2013), impidiendo la generación de políticas públicas acordes a las necesidades de esta actividad.

Lo anterior, junto con la insuficiente información existente (Zamora-Martínez y Nieto de Pascual-Pola 1995, Garibay-Orijel et al. 2009), falta de organización formal, acceso a servicios profesionales, recursos para inversión y su desarticulación con otros sectores (Mariaca et al. 2001, Martínez-Carrera et al. 2007), provoca un alto riesgo de agotar este recurso por la sobreexplotación (Ruiz et al. 2004). Además, la afectación del hábitat natural donde se encuentran, generada por la conversión a actividades agrícolas y ganaderas, que no siempre redundan en un mayor beneficio social o económico (Arteaga y Moreno 2006), comprometen su permanencia y causan efectos económicos, sociales y ambientales no deseables a largo plazo.

Por otro lado, la confirmación científica de las propiedades funcionales y medicinales de un gran número de hongos silvestres comestibles, así como el descubrimiento de sus bioactivos, se constituyen como la más reciente fortaleza para su domesticación y consecuente conservación y manejo del bosque (Martínez-Carrera et al. 2010). Su valor nutracéutico incluye propiedades anticancerígenas, antibióticas (antimicrobianas, antivirales, antibacterianas y antiparasitarias), antioxidantes, reductoras del nivel de colesterol y la hipertensión, antidiabéticas, así como para potenciar el sistema inmunológico humano (Jiménez et al. 2013, Méndez-Espinoza et al. 2013).

En México se conocen alrededor de 70 especies que han sido utilizadas en prácticas de medicina tradicional para el tratamiento de 40 tipos de problemas de salud humana (Guzmán 2008), utilizadas como tratamiento alternativo o como parte de la dieta (Jiménez et al. 2013, Méndez-Espinoza et al. 2013). Una de las más importantes es Cantharellus cibarius conocida tradicionalmente como “duraznito”, cuyas concentraciones de bioactivos (fenoles, 
flavonoides y ácido ascórbico) le confieren eficacia antioxidante y antimicrobiana, por lo que tiene un alto valor terapéutico (Ramesh y Pattar 2010, Jiménez et al. 2013, Méndez-Espinoza et al. 2013). Algunos otros ejemplos de hongos silvestres comestibles con potencial medicinal en México se enlistan en el cuadro 2.

Adicionalmente se ha reconocido el enorme potencial de los hongos silvestres comestibles como una fuente natural para el combate de la diabetes, la cual es un problema de salud pública en México, por lo que sus bioactivos naturales (polisacáridos, fibras, proteínas, etc.) pueden ser utilizados para la prevención, control y tratamiento de esta y otras enfermedades (De Silva et al. 2012, Kosanic et al. 2012, Jiménez et al. 2013), por lo que estos PFNM nativos tienen gran potencial para incorporarse en el corto plazo a la producción comercial de los hongos comestibles, funcionales y medicinales en México (Martínez-Carrera et al. 2010).

No obstante, es necesaria una mayor investigación de las propiedades, potencial e importancia de este recurso, ya que mucha de la información relacionada con estos PFNM se encuentra en riesgo de desaparecer (Vázquez 2012), ya que el campo mexicano está sufriendo de un fenómeno de migración (Burrola-Aguilar et al. 2012), envejecimiento rural, perturbación y alteración de zonas forestales (urbanización, cambio de uso del suelo, deforestación, etc.) que están generado una pérdida del conocimiento tradicional por el abandono de las actividades rurales, además de una transición de los hábitos alimentarios debido a fenómenos de aculturación (Ruan-Soto et al. 2013).

Con base en los elementos expuestos, la domesticación de los hongos silvestres comestibles debe realizarse considerando que este recurso es parte inseparable del ecosistema forestal y del sistema sociocultural de las comunidades indígenas y rurales de México. Por tanto, es necesario construir un entendimiento de cada especie en particular, el medio que los rodea y sus interacciones, con especial atención al manejo empírico y su integración a los agroecosistemas tradicionales, para establecer los principios de propagación y manejo bajo condiciones naturales. Lo anterior puede, en un futuro no muy lejano, dar pie a su cultivo in vitro o semicultivo in situ (Alvarado y Benítez 2009). Adicionalmente, el manejo de los hongos silvestres comestibles podría contribuir a las economías locales y ser un factor de transformación social de enorme relevancia en los ecosistemas forestales (Frutos et al. 2009 Sánchez y Mata 2012).

Cuadro 2. Hongos silvestres comestibles con potencial medicinal.

Edible wild mushrooms with medicinal potential.

\begin{tabular}{|c|c|c|c|}
\hline Especies & Nombres comunes & Propiedades medicinales & Referencias \\
\hline Agaricus subrufescens (Kauffman) & Sanjuanero & Antidiabético & De Silva et al. (2012) \\
\hline Hotson et Stuntz & & Antibacterial & Vázquez (2012) \\
\hline Agaricus campestris Schwein & & Antitumoral & Mata y Savoie (2012) \\
\hline \multirow[t]{3}{*}{ Amanita caesarea (Scop) Pers. } & \multirow[t]{3}{*}{ Hongo rojo Tecomate } & Antioxidante & \multirow{3}{*}{$\begin{array}{l}\text { Jiménez et al. (2013) } \\
\text { Bonet et al. (2010) }\end{array}$} \\
\hline & & Antibacteriano & \\
\hline & & Antiinflamatorio & \\
\hline Boletus edulis Bull. & Pancitas & Antioxidante & Bonet et al. (2010) \\
\hline Boletus pinicola Rea. & Cemitas & Antimicrobiano & Guzmán (2008) \\
\hline Boletus aestivalis (Paulet) Fr. & & & Kosanic et al. (2012) \\
\hline \multirow[t]{2}{*}{ Calvatia cyathiformis (Bosc.) Morgan } & Pedo de muerto & Cicatrizante & \multirow[t]{2}{*}{ Vázquez (2012) } \\
\hline & & Febrífugo & \\
\hline Cantharellus cibarius Fr. & Duraznito & Antioxidante & Ramesh y Pattar (2010) \\
\hline \multirow{6}{*}{ Cantharellus tubaeformis Fr. } & Flor de calabaza & Antimicrobiano & Jiménez et al. (2013) \\
\hline & Amarillito & Antitumoral & Méndez-Espinoza et al. (2013) \\
\hline & Chimequito & Antibacteriano & Bonet et al. (2010) \\
\hline & & Infecciones & \\
\hline & & gastrointestinales & \\
\hline & & Mejora la vista & \\
\hline Ganoderma lucidum (Curt:Fr) P. Karst & Repisas & Antidiabético & De Silva et al. (2012) \\
\hline \multirow{2}{*}{ Ganoderma applanatum (Pers) Pat. } & & Anti tiñas & Vázquez (2012) \\
\hline & & & Martínez-Carrera et al. (2010) \\
\hline
\end{tabular}




\begin{tabular}{|c|c|c|c|}
\hline Hydnum repandum L. & $\begin{array}{l}\text { Lengua de vaca } \\
\text { Gamuza } \\
\text { Lengua de gato }\end{array}$ & $\begin{array}{l}\text { Antioxidante } \\
\text { Antibacteriano } \\
\text { Antitumoral } \\
\text { Dolor de cabeza }\end{array}$ & Jiménez et al. (2013) \\
\hline Hypomyces lactifluorum (Schwein) Tul. & Enchilado & Antitumoral & $\begin{array}{l}\text { Guzmán (2008) } \\
\text { Jiménez et al. (2013) }\end{array}$ \\
\hline $\begin{array}{l}\text { Laccaria laccata (Scop.) Cooke } \\
\text { Laccaria amethystine (Huds) Cooke }\end{array}$ & Hongo de pajarito & Antitumoral & $\begin{array}{l}\text { Guzmán (2008) } \\
\text { Jiménez et al. (2013) }\end{array}$ \\
\hline Lactarius volemus (Fr.) Fr. & Hongo de leche & $\begin{array}{l}\text { Antioxidante } \\
\text { Antibacteriano } \\
\text { Antitumoral } \\
\text { Estimulante } \\
\text { Control de la presión baja }\end{array}$ & $\begin{array}{l}\text { Jiménez et al. (2013) } \\
\text { Vázquez (2012) }\end{array}$ \\
\hline Lentinula boryana (Berk et Mont) Pegler & Hongo de encino & Antitumoral & $\begin{array}{l}\text { Vázquez (2012) } \\
\text { Martínez-Carrera et al. (2010) }\end{array}$ \\
\hline Lepista nuda (Bull.) Cooke & Hongo moradito & $\begin{array}{l}\text { Antioxidante } \\
\text { Antitumoral } \\
\text { Tratamiento de lumbalgia } \\
\text { Esquelalgia }\end{array}$ & $\begin{array}{l}\text { Guzmán } 2008 \\
\text { Jiménez et al. (2013) }\end{array}$ \\
\hline Lycoperdon perlatum Pers & $\begin{array}{l}\text { Bombón } \\
\text { Huevito }\end{array}$ & $\begin{array}{l}\text { Cicatrizante } \\
\text { Antioxidante } \\
\text { Antimicrobiano }\end{array}$ & $\begin{array}{l}\text { Vázquez (2012) } \\
\text { Ramesh y Pattar (2010) }\end{array}$ \\
\hline Marasmius oreades (Bolton) Fr. & Hongo de corralito & $\begin{array}{l}\text { Antitumoral } \\
\text { Antioxidante } \\
\text { Antimicrobiano } \\
\text { Tonificante del estómago } \\
\text { Tratamiento de bronquitis }\end{array}$ & $\begin{array}{l}\text { Jiménez et al. (2013) } \\
\text { Ramesh y Pattar (2010) }\end{array}$ \\
\hline $\begin{array}{l}\text { Ramaria aurea (Schaeff) Quél } \\
\text { Ramaria araiospora Marr et Stuntz } \\
\text { Ramaria fennica (P. Karst) Ricken } \\
\text { Ramaria pallida (Schaeff) Ricken } \\
\text { Ramaria suecica (Fr.) Donk } \\
\text { Ramaria rubrievanescens Marr et Stuntz } \\
\text { Ramaria formosa (Pers.) Quél } \\
\text { Ramaria flava (Schaeff) Quél }\end{array}$ & $\begin{array}{l}\text { Pechuga } \\
\text { Escobeta } \\
\text { Pata de pájaro }\end{array}$ & $\begin{array}{l}\text { Antitumoral } \\
\text { Antioxidante } \\
\text { Antimicrobiana } \\
\text { Anticancerígena } \\
\text { Tonificante del estómago } \\
\text { Tratamiento de bronquitis }\end{array}$ & $\begin{array}{l}\text { Jiménez et al. (2013) } \\
\text { Montoya y Orrego (2012) } \\
\text { Aguilar-Cruz y Villegas } \\
\text { (2010) } \\
\text { Guzmán (2008) } \\
\text { Ramesh y Pattar (2010) }\end{array}$ \\
\hline Rusula cyanoxantha (Schaeff ex Schw) Fr. & $\begin{array}{l}\text { Trompa } \\
\text { Duraznito }\end{array}$ & $\begin{array}{l}\text { Antitumoral } \\
\text { Estimulante }\end{array}$ & Vázquez (2012) \\
\hline Schizophyllum commune Fr- & $\begin{array}{l}\text { Oreja de palo } \\
\text { Mulato }\end{array}$ & Antitumoral & Vázquez (2012) \\
\hline Tricholoma magnivelare (Peck) Redhead & $\begin{array}{l}\text { Hongo blanco } \\
\text { Matsutake }\end{array}$ & $\begin{array}{l}\text { Antiinflamatorio Antitu- } \\
\text { moral } \\
\text { Tratamiento de la } \\
\text { hepatopatía }\end{array}$ & $\begin{array}{l}\text { Bonet et al. (2010) } \\
\text { Jiménez et al. (2013) }\end{array}$ \\
\hline Tylopilus felleus (Bull) P. Karst & Lengua de toro & $\begin{array}{l}\text { Antiinflamatorio } \\
\text { Antitumoral } \\
\text { Tratamiento de hepato- } \\
\text { patía }\end{array}$ & Jiménez et al. (2013) \\
\hline
\end{tabular}




\section{CONCLUSIONES}

El potencial de utilización de diversas especies de hongos silvestres comestibles como fuente de alimento, ingresos y conservación del entorno ecológico, como un factor de transformación social en áreas rurales es enorme. Sin embargo, los estudios ecológicos y biotecnológicos de este importante recurso, a pesar de su gran relevancia, se encuentran en etapas muy tempranas, son puntuales y la información se encuentra dispersa. Esto pone en evidencia la importancia de recopilar, sistematizar, resguardar y difundir el conocimiento etnomicológico que existe en México, que es la base para generar procesos de domesticación, cultivo y aprovechamiento. No obstante, este debe realizarse con un enfoque interdisciplinario y considerando todos los componentes que integran la recolección de estos hongos, especialmente su interacción con el bosque. El reconocimiento de la domesticación, desde este punto de vista, contribuiría a valorar la diversidad de este recurso, dar impulso a un modelo productivo que favorezca el equilibrio entre conservación y desarrollo, así como la creación de nuevas alternativas productivas que sean competitivas y sostenibles en un esquema de mercado global.

\section{REFERENCIAS}

Abbo S, S Lev-Yadun, A Gopher. 2012. Plant Domestication and Crop Evolution in the Near East: On Events and Processes. Critical Reviews in Plant Sciences 31(3): 241-257. DOI: 10.1080/07352689.2011.645428.

Aguilar-Cruz Y, M Villegas. 2010. Especies de Gomphales comestibles en el municipio de Villa del Carbón Estado de México. Revista Mexicana de Micología 31: 1-8.

Alvarado G, G Benítez. 2009. El enfoque de agroecosistemas como una forma de intervención científica en la recolección de hongos silvestres comestibles. Tropical and Subtropical Agroecosystems 10: 531-539.

Andrade GRH, G Mata, JE Sánchez. 2012. La producción iberoamericana de hongos comestibles en el contexto internacional. In Mata G, JE Sánchez eds. Hongos comestibles y medicinales en Iberoamérica: investigación y desarrollo en un entorno multicultural. Chiapas, México. El Colegio de la Frontera Sur. p. 9-18.

Arteaga MB, ZC Moreno. 2006. Los hongos comestibles silvestres de Santa Catarina del Monte, Estado de México. Revista Chapingo Serie Ciencias Forestales y del Ambiente 12(2): 125-131.

Bell M, JC Walker. 1992. Late quaternary environmental change: physical and human perspectives. London, UK. Longman Scientific and Technical. 273 p.

Benítez-Badillo G, G Alvarado-Castillo, ME Nava-Tablada, A Pérez-Vázquez. 2013. Análisis del marco regulatorio en el aprovechamiento de los hongos silvestres comestibles en México. Revista Chapingo Serie Ciencias Forestales y del Ambiente 19(3): 363-374. DOI: 10.5154/r.rchscfa.2012.09.055.

Boa E. 2004. Wild edible fungi: a global overview of their use and importance to people. Rome, Italy. FAO. 147 p.

Bonet JA, M Palahí, C Colinas, T Pukkala, CR Fischer, J Miina,
J Martínez de Aragón. 2010. Modelling the production and species richness of wild mushrooms in pine forests of the Central Pyrenees in northeastern Spain. Canadian Journal of Forest Research 40(2): 347-356.

Bonet JA, S De-Miguel, J Martínez de Aragón, T Pukala, M Palahí. 2012. Immediate effect of thinning on the yield of Lactarius group deliciosus in Pinus pinaster forests in Northeastern Spain. Forest Ecology and Management 265: 211-217.

Burrola-Aguilar C, O Montiel, R Garibay-Orijel, L Zizumbo-Villareal. 2012. Conocimiento tradicional y aprovechamiento de los hongos comestibles en la región de Amanalco, Estado de México. Revista Mexicana de Micología 35: 1-16.

Burrola-Aguilar C, R Garibay-Orijel, A Argüelles-Moyao. 2013. Abies religiosa forests harbor the highest species density and sporocarp productivity of wild edible mushrooms among five different vegetation types in a neotropical temperate forest region. Agroforestry Systems 87: 1101-1115. DOI: 10.1007/s10457-013-9623-z.

Castillo-Guevara C, C Lara, G Pérez. 2012. Micofagia por roedores en un bosque templado del centro de México. Revista Mexicana de Biodiversidad 83: 772-777. DOI: 10.7550/ rmb. 27445

Chang ST, PG Miles. 2004. Mushroom cultivation, nutritional value, medicinal effect, and environmental impact. Washington D.C., USA. CRC Press. 451 p.

Conferencia ministerial de Helsinki (Finlandia). 1993. Resolución de Helsinki No 1: Directrices generales para una gestión sostenible de los bosques en Europa. Consultado 7 ene. 2014. Disponible en http://www.magrama.gob.es/es/ biodiversidad/temas/politica-forestal/forest-europe/fe_conferencias_ministeriales.aspx\#para4.

Danell E, FJ Camacho. 1997. Successful cultivation of the golden chanterelle. Nature 385: 303. DOI: 10.1038/385303a0.

De Silva DD, S Rapior, KD Hyde, AH Bahkali. 2012. Medicinal mushrooms in prevention and control of diabetes mellitus. Fungal Diversity 56: 1-29.

Egli S. 2011. Mycorrhizal mushroom diversity and productivity-an indicator of forest health? Annals of Forest Science 68(1): 81-88. DOI: 10.1007/s13595-010-0009-3.

Estrada-Martínez E, G Guzmán, TD Cibrián, PR Ortega. 2009. Contribución al conocimiento etnomicológico de los hongos silvestres comestibles en los mercados regionales y comunidades de la sierra nevada (México). Interciencia 34: 25-33.

Fernández-Toirán LM, T Ágreda, JM Olano. 2006. Stand age and sampling year effect on the fungal fruit body community in Pinus pinaster forest in central Spain. Canadian Journal of Botany 84: 1249-1258. DOI: 10.1139/b06-087.

Frutos P, F Martínez-Peña, P Ortega-Martínez, S Esteban. 2009. Estimating the social benefits of recreational harvesting of edible wild mushrooms using travel cost methods. Forest Systems 18(3): 235-246. DOI: http://dx.doi.org/10.5424/ fs/2009183-01065.

Garibay-Orijel R, A Ramírez-Terrazo, M Ordaz-Velázquez. 2012. Women care about local knowledge, experiences from ethnomycology. Journal of Ethnobiology and Ethnomedicine 8(25): 1-12. DOI: 10.1186/1746-4269-8-25.

Garibay-Orijel R, J Córdova, J Cifuentes, R Valenzuela, A Estrada-Torres, A Kong. 2009. Integrating wild mushrooms use into a model of sustainable management for indigenous 
community forests. Forest Ecology and Management 258: 122-131. DOI: 10.1016/j.foreco.2009.03.051.

Guzmán G. 1994. Los hongos en la medicina tradicional de Mesoamérica y de México. Revista Iberoamericana de Micología 11: 81-85.

Guzmán G. 2008. Diversity and use of traditional Mexican medicinal fungi. A review. International Journal Medicinal Mushrooms 10: 209-217.

Hernández XE. 1993. Aspects of plant domestication in México: a personal view. In Ramamoorthy TP, R Bye, A Lot, J Fa eds. Biological diversity of México: origins and distribution. Oxford University Press. p. 733-753.

Iwase K. 1997. Cultivation of mycorrhizal mushroom. Food Reviews International 13: 431-442. DOI: 10.1080/87559129709541130.

Jakuba T, J Kottferová, J Mareková, M Ondrasovic, O Ondrasovicová. 2008. Ecology and domestication. Folia Veterinaria 52: $83-84$

Jiménez RM, J Pérez-Moreno, JJ Almaraz-Suárez, M TorresAquino. 2013. Hongos silvestres con potencial nutricional, medicinal y biotecnológico comercializados en Valles Centrales, Oaxaca. Revista Mexicana de Ciencias Agrícolas 4(2): 199-213.

Kosanic M, B Rankovic, M Dasic. 2012. Mushrooms as possible antioxidant and antimicrobial agents. Iranian Journal of Pharmaceutical Research 11(4): 1095-1102.

Krapovickas A. 2011. Sembrar, plantar, cultivar, domesticar. Bonplandia 20(2): 419-426.

Kües U, Y Liu. 2000. Fruiting body production in basidiomycetes. Applied Microbiolology Biotechnology 54: 414-152. DOI: $10.1007 / \mathrm{s} 002530000396$.

Lara-Vázquez F, AT Romero-Contreras, C Burrola-Aguilar. 2013. Conocimiento tradicional sobre los hongos silvestres en la comunidad otomí de San Pedro Arriba; Temoaya, Estado de México. Agricultura, Sociedad y Desarrollo 10(3): 305-333.

Mariaca MR, PL Silva, MC Castaños. 2001. Proceso de recolección y comercialización de hongos comestibles silvestres en el valle de Toluca, México. Ciencia Ergo Sum 8: 30-40.

Martínez de Aragón J, P Riera, M Giergiczny, C Colinas. 2011. Value of wild mushroom picking as an environmental service. Forest Policy and Management 252(1-3): 239-256. DOI: 10.1016/j.forpol.2011.05.003.

Martínez-Carrera D, N Curvetto, M Sobal, P Morales, VM Mora. 2010. Hacia un desarrollo sostenible del sistema de producción-consumo de los hongos comestibles y medicinales en Latinoamérica: Avances y perspectivas en el siglo XXI. Puebla, México. Red Latinoamericana de Hongos Comestibles y Medicinales-COLPOS-UNS-CONACYT-AMCUAEM-UPAEP-IMINAP. 648 p.

Martínez-Carrera D, P Morales, M Sobal, M Bonilla, W Martínez. 2007. México ante la globalización en el siglo XXI: El sistema de producción consumo de los hongos comestibles. In Sánchez JE, D Martínez-Carrera, G Mata, H Leal eds. El Cultivo de Setas Pleurotus spp en México. Chiapas, México. El Colegio de la Frontera Sur. p. 209-224.

Martínez-Peña F, S de-Miguel, T Pukkala, JA Bonet, P OrtegaMartínez, J Aldea, J Martínez de Aragón. 2012. Yield models for ectomycorrhizal mushrooms in Pinus sylvestris forests with special focus on Boletus edulis and Lactarius group deliciosus. Forest Ecology and Management 282: 63-69.
Mata G, JM Savoie. 2012. Agaricus subrufescens un hongo comestible y medicinal de gran potencial en México. In Mata G, JE Sánchez eds. Hongos comestibles y medicinales en Iberoamérica: investigación y desarrollo en un entorno multicultural. Chiapas, México. El Colegio de la Frontera Sur. p. 137-144.

Méndez-Espinoza C, E García-Nieto, AM Esquivel, MM González, EV Bautista, CC Ezquerro, LJ Santacruz. 2013. Antigenotoxic potential of aqueous extracts from the chanterelle mushroom, Cantharellus cibarius (higher Basidiomycetes), on human mononuclear cell cultures. International Journal of Medicinal Mushrooms 15(3): 325-32.

Meyer RS, AE DuVal, HR Jensen. 2012. Patterns and processes in crop domestication: an historical review and quantitative analysis of 203 global food crops. New Phytologist 196: 29-48. DOI: 10.1111/j.1469-8137.2012.04253.x

Montoya A, N Hernández, C Mapes, A Kong, A Estrada-Torres. 2008. The Collection and Sale of Wild Mushrooms in a Community of Tlaxcala, Mexico. Economic Botany 62(3): 413-424. DOI: 10.1007/s12231-008-9021-z

Montoya S, CE Orrego. 2012. Growth, fruiting and lignocellulolytic enzyme production by the edible mushroom Grifolafrondosa (maitake). World Journal of Microbiology and Biotechnology 28(4): 1533-1541.

Morcillo M, M Sánchez. 2004. ¿Por qué es tan difícil cultivar hongos micorrízicos comestibles? Terralia 45: 80-85.

Ortega-Martínez P, F Martínez-Peña. 2008. A sampling method for estimating sporocarps production of wild edible mushrooms of social and economic interest. Investigación agraria. Sistemas y recursos forestales 17(3): 228-237.

Pastor BJF. 2002. Los productos forestales no maderables, una fuente de materia prima para el desarrollo de la industria eléctrica en Cuba. Revista Chapingo serie Ciencias Forestales y del Ambiente 8(2): 147-152.

Pérez-Moreno J, A Lorenzana-Fernández, V Carrasco-Hernández, A Yescas-Pérez. 2010. Los hongos comestibles silvestres del parque nacional Izta-Popo, Zoquiapan y anexos. Montecillo, Texcoco, Estado de México. Colegio de Postgraduados, SEMARNAT, CONACYT. 167 p.

Pérez-Moreno J, M Martínez-Reyes, A Yescas-Pérez, A DelgadoAlvarado, B Xoconostle-Cázares. 2008. Wild Mushroom Markets in Central Mexico and a Case Study at Ozumba. Economic Botany 62(3): 425-436. DOI: 10.1007/s12231008-9043-6.

Pérez-Moreno J. 2012. Los hongos comestibles ectomicorrizicos y su biotecnología. In Mata G, JE Sánchez eds. Hongos comestibles y medicinales en Iberoamérica: investigación y desarrollo en un entorno multicultural. Chiapas, México. El Colegio de la Frontera Sur. p. 19-28.

Ramesh Ch, MG Pattar. 2010. Antimicrobial properties, antioxidant activity and bioactive compounds from six wild edible mushrooms of western ghats of Karnataka, India. Pharmacognosy Research 2: 107-12. DOI: 10.4103/09748490.62953.

Reyna DS. 2012a. Sostenibilidad de la Truficultura: aspectos ecológicos, económicos y sociales. In Reyna DS ed. Truficultura. Fundamentos y Técnicas. Madrid, España. Mundiprensa. p. 49-72.

Reyna DS. 2012b. Introducción. Historia y Perspectivas de la Truficultura. In Reyna DS ed. Truficultura. Fundamentos y Técnicas. Madrid, España. Mundiprensa. p. 25-48. 
Reyna S, S Garcia-Barreda. 2014. Black truffle cultivation: a global reality. Forest Systems 23(2): 317-328. DOI: http:// dx.doi.org/10.5424/fs/2014232-04771.

Ruan-Soto F, J Caballero, C Martorell, J Cifuentes, AR GonzálezEsquinca, R Garibay-Orijel. 2013. Evaluation of the degree of mycophilia-mycophobia among highland and lowland inhabitants from Chiapas, Mexico. Journal of Ethnobiology and Ethnomedicine 9(36): 1-13. DOI: 10.1186/1746-42699-36.

Ruan-Soto F, J Cifuentes, R Mariaca, F Limón, L Pérez-Ramírez, S Sierra. 2009. Uso y manejo de hongos silvestres en dos comunidades de la Selva Lacandona, Chiapas, México. Revista Mexicana de Micología 29: 61-72.

Ruiz PM, B Belcher, R Achdiawan, M Alexaides, C Aubertin, J Caballero, B Campbell, C Clement, T Cunningham, A Fantini, H de Foresta, C García-Fernández, KH Gautam, P Hersch-Martínez, W de Jong, K Kusters, MG Kutty, C López, M Fu, MA Martínez-Alfaro, TR Fair, O Ndoye, R Ocampo, N Rai, M Ricker, K Schereckenberg, S Shackleton, P Shanley, T Sunderland, Y Youn. 2004. Markets drive the specialization strategies of forest peoples. Ecology and Society 9: 1-29.

Sánchez JE, G Mata. 2012. Cultivo y aprovechamiento de macromicetos. Una tendencia global en crecimiento. In Mata G, JE Sánchez eds. Hongos comestibles y medicinales en Iberoamérica: investigación y desarrollo en un entorno multicultural. Chiapas, México. El Colegio de la Frontera Sur. p. 365-376.

Sangabriel-Conde W, D Trejo-Aguilar, A Soto-Estrada, R Ferrera-Cerrato, L Lara-Capistrán. 2010. Potencial de colonización de hongos micorrícico-arbusculares en suelos cultivados con papayo bajo diferentes manejos de producción. Revista Mexicana de Micología 31: 45-52.

Savoie JM, ML Largeteau. 2011. Production of edible mushrooms in forests: trends in development of a mycosilviculture. Applied Microbiology and Biotechnology 89: 971-
979. DOI: $10.1007 / \mathrm{s} 00253-010-3022-4$

Sistema Nacional de Información Forestal. 2012. Inventario Nacional Forestal y de Suelos, Informe de resultados 2004-2009. Consultado 9 ene. 2014. Disponible en http:// www.cnf.gob.mx:8080/snif/portal/infys/temas/resultados-2004-2009

Smith SE, D Read. 2008. Mycorrhizal Symbiosis. London, UK. Elseiver. 800 p.

Solbrig O. 2004. Ventajas y desventajas de la agrobiotecnología. In Bárcena A, A Katz, J Morales, M Schaper eds. Los transgénicos en América Latina y el Caribe: un debate abierto. Santiago, Chile. CEPAL. p. 33-69.

Tacón A, J Palma. 2006. La comercialización de los productos forestales no madereros: una oportunidad para el manejo comunitario y la valoración del bosque nativo. Revista Bosque Nativo 39: 253-266.

Vázquez SM. 2012. Macromicetos medicinales provenientes de la sierra norte de Puebla, México; depositados en el Herbario “Gastón Guzmán”, ENCB-IPN. Etnobiología 10(2): 34-37.

Villareal RL, J Pérez-Moreno. 1989. Los hongos comestibles silvestres de México, un enfoque integral. Micología Neotropical Aplicada 2: 77-114.

Wang Y, IR Hall. 2004. Edible ectomycorrhizal mushrooms: challenges and achievements. Canadian Journal of Botany 82: 1063-1073. DOI: 10.1139/B04-051.

Wilsey D. 2006. Can NTFP certification help to ensure the viability of natural production in community-managed extractive reserves? Consultado 23 abr. 2013. Disponible en http://abstracts.co.allenpress.com/pweb/esai2006/document/59051.

Zamora-Martínez MC, C Nieto de Pascual-Pola. 1995. Natural production of wild edible mushrooms in the southwestern rural territory of Mexico City, Mexico. Forest Ecology and Management 72(1): 13-20. DOI: http://dx.doi. org/10.1016/0378-1127(94)03450-B. 
